\title{
Center of gravity transfer velocity during sit-to-stand is closely related to physical functions regarding fall experience of the elderly living in community dwelling
}

\author{
Takayoshi Yamada $^{1^{*}}$, Shinichi Demura ${ }^{2}$, Kenji Takahashi $^{3}$ \\ ${ }^{1}$ Faculty of Education and Regional studies, University of Fukui, Fukui, Japan; \\ *Corresponding Author: yamadat@u-fukui.ac.jp \\ ${ }^{2}$ Graduate School of Natural Science \& Technology, Kanazawa University, Ishikawa, Japan \\ ${ }^{3}$ Faculty of Community Health Care, Teikyo Heisei University, Chiba, Japan
}

Received 22 October 2013; revised 26 November 2013; accepted 4 December 2013

Copyright (C) 2013 Takayoshi Yamada et al. This is an open access article distributed under the Creative Commons Attribution License, which permits unrestricted use, distribution, and reproduction in any medium, provided the original work is properly cited.

\section{ABSTRACT}

This study examines the difference of the center of gravity (CG) velocity during sit-to-stand (STS) movement between the elderly with and without fall experience. Fifteen elderly without fall experience (age: $75.7 \pm 4.8 \mathrm{yr}$ ), with one fall experience (age: $75.7 \pm 5.0 \mathrm{yr}$ ), and two or more fall experiences (age: $75.7 \pm 4.8 \mathrm{yr}$ ) participated in the measurement of $10 \mathrm{~m}$ maximal walking speed, functional reach, one-legged standing duration with vision, maximal isometric knee extension, hip flexion, ankle extension and toe flexion muscle strengths, fall risk assessment and ADL questionnaire, and CG velocity during STS movement. No significant differences were found in muscle strength, walking and balance tests ( $F<$ $2.7, p>0.078)$. The group with two or more fall experiences was significantly higher than the other groups in the fall risk assessment, and lower than the group without fall experience in ADL $(F=8.5$ and $6.1, p=0.001$ and 0.005$)$. Meanwhile, significant differences were found in the maximal and mean CG velocity during STS movement among all groups. The group without fall experience was the greatest, and the group with fall experience was less than half $(F=9.0$ and 11.1, $p=0.001$ and $<0.001$ ). In conclusion, CG velocity during STS movement has a closer relationship with fall risk than leg strength, and walking and balance tests.

Keywords: Sit-to-Stand Movement; Physical

\section{Function; Fall}

\section{INTRODUCTION}

Fall incidence rate in the elderly is approximately $30 \%$ in developed nations [1,2]. Approximately $20 \%$ elderly Japanese experience at least one fall annually [3-7]. Falls markedly decrease the degree of independence because they cause fractures and post-fall syndrome; they are a major cause of the elderly becoming bedridden and requiring nursing care. Approximately $10 \%$ elderly fall and experience fractures [8], thus increasing hospitalization and mortality rates in the elderly because of post-fall syndrome $[9,10]$. This trend is particularly significant in elderly aged $\geq 85$ years [11]. Since 1987 to date, the number of transcervical fractures has increased from 53,200 to 117,900 [12]. Because the elderly population is predicted to increase, the number of falls and serious fall injuries will probably increase; the number of elderly requiring nursing care or becoming bedridden will also increase. Therefore, fall prevention measures are important for maintaining and enhancing the quality of life and for preventing the requirement for nursing care or becoming bedridden because of post-fall syndrome.

The American Geriatric Society [13] guidelines for prevention of falls in older persons state that muscle weakness (4.4), fall history (3.0), gait deficit (2.9), balance deficit (2.9), use of assistive devices (2.6), visual deficits (2.5), arthritis (2.4), impaired activities of daily living (ADL) (2.3), depression (2.2), cognitive impairment (1.8) and age $>80$ years (1.7) are factors closely related to increased fall risk in the elderly (odds ratios indicated in parentheses). Falling is a multiple risk factor 
syndrome comprising internal (i.e., disease and physical function) and external (i.e., bump and slippery floor) factors. However, decrease in physical functioning, such as muscle weakness and gait and balance deficits, has a significant influence. Therefore, to prevent falls, adequate measurement and evaluation of physical function related to falling and maintaining proper physical function are required. Several fall risk factors related to muscle strength and gait and balance functions have been reported; associated batteries of tests have been developed. However, it may be useful to evaluate multiple risk factors simultaneously rather than separately because falling is a multiple risk-factor syndrome. Therefore, a more valid evaluation would include the relationships among multiple factors.

The goal is to easily measure and evaluate physical functioning in the elderly using ADL and to present the results to the elderly in an understandable way. Physical function tests using daily life activities have been proposed, such as gait and walking up/down stairs. However, because the sit-to-stand (STS) movement precedes all daily life activities [14], the ability to perform a smooth and stable STS movement is indispensable for the elderly to maintain an independent life [15]. Therefore, the importance of evaluating the ability to achieve STS has been proposed and evaluation tests have been developed. STS is an upward movement that transfers the center of gravity [16,17]; it is divided into trunk flexion and trunk and knee extension phases. The former describes the phase between trunk flexion, with forward (horizontal) transfer of the center of gravity from the initiation of movement until the hip lifts from the seat. The latter describes the phase between hip lift-off and a standing posture, with hips and both knee joints extended. A stable STS movement requires a smooth transfer of the center of gravity, which differs between the former and latter phases. Leg strength and balance ability during the center of gravity transfer are vital. The physical functions cited above as fall risk factors are intricately involved in STS movement. Thus, physical functions closely related to falls may be evaluated by measuring STS movement.

This study examined the velocity at which the center of gravity is transferred during STS movement in the elderly with and without fall experience.

\section{METHODS}

\subsection{Subjects}

The purpose and significance of this study were explained to all subjects in a community activity support project for preventing primary nursing care in the primary prevention project for the elderly, which was organized by the local government. Fifteen elderly subjects were divided into three groups: without (age, $75.7 \pm 4.8$ years; height, $149.1 \pm 5.8 \mathrm{~cm}$; body mass, $53.0 \pm 6.4 \mathrm{~kg}$ ), with one (age, $75.7 \pm 5.0$ years; height, $149.2 \pm 5.4 \mathrm{~cm}$; body mass, $51.6 \pm 8.4 \mathrm{~kg}$; number of fall experiences, 1.0 \pm 0.0 /year), and with more than two fall experiences (age, $75.7 \pm 4.8$ years; height, $149.5 \pm 5.3 \mathrm{~cm}$; body mass, 55.9 $\pm 6.3 \mathrm{~kg}$, number of fall experiences, $2.2 \pm 0.4$ /year). After considering the age and physical characteristics of the elderly, only subjects who gave their consent were selected. All subjects were living independently in a community dwelling. This study protocol was approved by the Ethics Committee on Human Experimentation of Faculty of Human Science, Kanazawa University (authorization number: 2012-04).

\subsection{Materials and Measurement Methods}

\subsubsection{Center of Gravity Transfer Velocity during STS Movement}

Figure 1 shows the experimental scheme. The center of gravity transfer velocity during STS movement was measured using FITRO Dyne Premium (FiTRONIC sro, Bratislava, Slovakia). This device measures the length of a pulled or returned cord from the bobbin, which works with a built-in rotary encoder. As shown in Figure 1, the subjects wore a belt around their waist. The cord was fixed at the left crista iliaca position on the belt, and the length of the pulled or returned cord was measured when each subject performed STS movement [18]. The center of gravity is located in the abdomen during a sitting posture and is transferred from the abdomen to the lumbar spine during movement to stabilize the lumbar spine [19]. Crista iliaca transfer velocity measured by the time change of the pulled or returned cord length was assumed to reflect the center of gravity transfer velocity during STS movement.

Subjects maintained a sitting posture, kept both legs a shoulder width apart with bare feet, stretched the trunk in a straight line, held a $90^{\circ}$ ankle angle, and crossed their arms in front of their chest. STS movement was con-

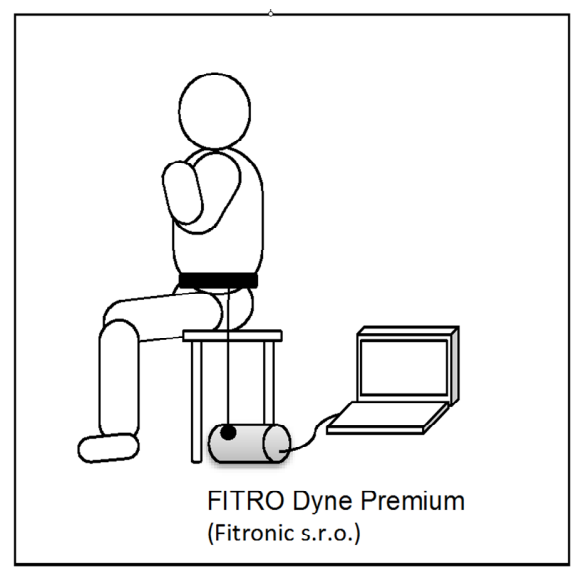

Figure 1. Experimental schema. 
ducted as rapidly as possible from the above posture after the tester's signal. A chair was adjusted to the knee height of each subject. Data were recorded every $1 / 100 \mathrm{~s}$ using a personal computer.

\subsubsection{Maximal Isometric Knee Extension, Hip Flexion, Ankle Extension and Toe Flexion Strength}

Maximum isometric knee extension and hip flexion strength was measured using a hand-held dynamometer ( $\mu$ Tas F-1, Anima Co., Ltd.). Subjects were instructed to extend or flex with maximum effort from a sitting posture with the knee or hip joint at $90^{\circ}$. Maximum isometric ankle extension strength was measured using an ankle extension muscle strength device (T.K.K. 3360; Takei Co., Ltd). Subjects were instructed to extend the ankle joint with maximum effort from a sitting posture with a fully extended knee joint and a $90^{\circ}$ flexed ankle joint. Isometric toe flexion strength was measured using a toe flexion muscle strength device (T.K.K. 3362; Takei Co., Ltd.). Subjects were instructed to flex their toes with maximum effort against a lever connected to a strain gauge.

\subsubsection{0-Meter Maximal Walking Speed}

Walking speed was measured while walking 10-meter with maximum effort on a flat floor without obstacles. The subjects began walking 3-meter before the start line and walked 3-meter past the finish line, thus walking a total of $16 \mathrm{~m}$.

\subsubsection{Functional Reach}

Functional reach was measured as outlined by Duncan et al. [20]. Each subject stood in line with a yardstick that was fixed on the wall at a dominant acromion height and adjusted to the tip of the middle finger of the dominant hand at the starting point of the yardstick. Subsequently, a tester measured the distance up to which the subject could maximally extend their fingertip in an upright posture.

\subsubsection{One-Legged Standing Duration with Vision}

One-legged standing duration with vision was measured as outlined by the Ministry of Education, Culture, Sports, Science and Technology in Japan. The maximum duration that the subjects could stand on one leg with their hands on their waist was measured. The measurement ended when a lifted leg contacted the floor, the supporting leg moved, one or both hands left the waist, or on completion of 120 seconds from the start.

\subsubsection{ADL Questionnaire from the Ministry of Education, Culture, Sports, Science and Technology and the Fall Risk Assessment Questionnaire}

Before the above stated functional tests, subjects re- sponded to the ADL questionnaire of the Ministry of Education, Culture, Sports, Science, and Technology, and the fall risk assessment questionnaire [21]. The ADL questionnaire comprises 12 items regarding daily life activities such as STS, gait, obstacle avoidance, balance, and wearing/removing clothes. The fall risk assessment questionnaire comprises eight factors comprising 15 items regarding gait and balance deficit, muscle weakness, fall risk due to disease and drugs, external factors related to a fall, watching/listening deficits, fear of falling, and ADL limitations. The questionnaires were individually completed by the subjects in "yes" or "no" responses.

\subsection{Procedure}

Upon entering the laboratory, subjects completed ADL and fall risk assessment questionnaires. Then, maximum isometric knee extension, hip flexion, ankle extension, and toe flexion strength; 10-meter maximum walking speed; functional reach; one-legged standing duration with vision; and center of gravity transfer velocity during STS movement were measured. All muscle strength tests were performed twice on the right and left legs, and sufficient rest was given between trials and items. All items except the questionnaires were measured twice with sufficient rest between trials.

\subsection{Parameters}

The total score of each ADL item and the number of high fall risk responses in the fall risk assessment questionnaire were calculated for analysis. The mean of the maximum right/left leg strength trial was calculated for analysis. The higher value from the two trials was selected for analysis in the functional reach and 10-meter maximum walking speed. Maximum and mean center of gravity transfer velocities between the start and completion of STS movement were selected with reference to a previous study [22], and a mean value from the two trials was used for analysis.

\subsection{Statistical Analysis}

Independent one-way analysis of variance (ANOVA) was used to examine the differences among the elderly without, with one, and with more than two experiences for all parameters selected in this study. Tukey's honestly significant difference test was used as a post-hoc. A $\mathrm{p}$ value $<0.05$ was considered statistically significant.

\section{RESULTS}

No significant differences in age, height, or body mass were observed among the groups $(\mathrm{F}<1.40, \mathrm{p}>0.258)$. A significant difference in the number of fall experiences 
was observed; subjects with more than two fall experiences were the maximum, whereas those without fall experience were the minimum $(\mathrm{F}=318.5, \mathrm{p}<0.05)$. Table 1 shows the results of independent one-way ANOVA results for ADL and fall risk scores, maximum isometric knee extension, hip flexion, ankle extension, toe flexion strength, 10-meter maximum walking speed, functional reach, one-legged standing duration with vision tests, and maximum and mean center of gravity transfer velocity. No significant difference in maximum walking speed, functional reach, maximum isometric knee extension, hip flexion, ankle extension, and toe flexion strength was observed. A significant difference in ADL and fall risk scores was observed. In the former, the scores of the the group without fall experience were greater than those of the group with more than two fall experiences; in the latter, the scores of the group with more than two experiences were greater than those of the other groups. Significant differences in scores were observed among all groups. The group without fall experience had the maximum scores, while that with more than two fall experiences had the minimum scores.

\section{DISCUSSION}

Several factors contribute to the fall incidence rate, but decrease in function, such as muscle weakness, gait deficits, balance deficits, and impaired ADL, is one of the most significant contributors [13]. It has been suggested that physical functions in the elderly with fall experi-

Table 1. Functional indices, maximal and mean center of gravity transferring velocities of the subjects, without, with once and more than twice fall experience.

\begin{tabular}{|c|c|c|c|c|c|c|c|}
\hline & \multirow{3}{*}{$\mathrm{n}$} & More than twice & Once & No fall & \multirow{3}{*}{$\mathrm{F}$} & \multirow{3}{*}{$\mathrm{p}$} & \multirow{3}{*}{ Post-hoc } \\
\hline & & (M) & $(\mathrm{O})$ & $(\mathrm{N})$ & & & \\
\hline & & 15 & 15 & 15 & & & \\
\hline \multirow{2}{*}{ Maximal walking speed $(\mathrm{cm} / \mathrm{s})$} & Mean & 1.6 & 1.6 & 1.7 & \multirow{2}{*}{0.2} & \multirow{2}{*}{0.850} & \\
\hline & SD & 0.4 & 0.3 & 0.2 & & & \\
\hline \multirow[b]{2}{*}{ Functional reach $(\mathrm{cm})$} & Mean & 30.4 & 29.9 & 30.2 & \multirow[b]{2}{*}{0.0} & \multirow[b]{2}{*}{0.963} & \\
\hline & SD & 6.7 & 5.7 & 4.3 & & & \\
\hline \multirow{2}{*}{ One-legged standing duration with vision (sec) } & Mean & 12.2 & 31.3 & 35.9 & \multirow{2}{*}{2.2} & \multirow{2}{*}{0.126} & \\
\hline & $\mathrm{SD}$ & 11.4 & 38.0 & 40.8 & & & \\
\hline \multirow[b]{2}{*}{ Maximal isometric knee extension strength $(\mathrm{kg})$} & Mean & 6.2 & 7.2 & 8.0 & \multirow[b]{2}{*}{2.7} & \multirow[b]{2}{*}{0.078} & \\
\hline & SD & 2.2 & 1.8 & 2.1 & & & \\
\hline \multirow{2}{*}{ Maximal isometric hip flexion strength (kg) } & Mean & 10.8 & 12.5 & 12.3 & \multirow{2}{*}{1.2} & \multirow{2}{*}{0.322} & \\
\hline & SD & 4.0 & 3.0 & 2.7 & & & \\
\hline \multirow[b]{2}{*}{ Maximal isometric ankle extension strength $(\mathrm{N})$} & Mean & 313.6 & 414.5 & 427.3 & \multirow[b]{2}{*}{2.3} & \multirow[b]{2}{*}{0.114} & \\
\hline & SD & 166.7 & 185.6 & 118.7 & & & \\
\hline \multirow{2}{*}{ Maximal isometric toe flexion strength $(\mathrm{kg})$} & Mean & 3.4 & 4.6 & 4.3 & \multirow{2}{*}{1.4} & \multirow{2}{*}{0.262} & \\
\hline & SD & 1.6 & 2.3 & 2.0 & & & \\
\hline \multirow{2}{*}{ Fall risk score (point) } & Mean & 5.4 & 3.5 & 2.7 & \multirow[b]{2}{*}{8.5} & \multirow[b]{2}{*}{0.001} & \multirow[b]{2}{*}{$\mathrm{M}>\mathrm{O}, \mathrm{N}$} \\
\hline & SD & 2.2 & 1.8 & 1.5 & & & \\
\hline \multirow{2}{*}{ ADL score (point) } & Mean & 21.5 & 25.0 & 27.1 & \multirow{2}{*}{6.1} & \multirow{2}{*}{0.005} & \multirow{2}{*}{$\mathrm{N}>\mathrm{M}$} \\
\hline & $\mathrm{SD}$ & 5.0 & 4.9 & 3.2 & & & \\
\hline & Mean & 71.9 & 81.2 & 101.5 & & & \\
\hline Maximal center of gravity transferrıng velocity $(\mathrm{cm} / \mathrm{s})$ & SD & 28.6 & 13.3 & 11.9 & 9.0 & 0.001 & $N>0>M$ \\
\hline & Mean & 38.0 & 45.3 & 56.8 & & & \\
\hline Mean center of gravity transferring velocity $(\mathrm{cm} / \mathrm{s})$ & SD & 15.5 & 7.4 & 8.2 & 11.1 & $<0.001$ & $\mathrm{~N}>\mathrm{O}>\mathrm{M}$ \\
\hline
\end{tabular}


ences are inferior to those in the elderly without fall experience; this impairment of physical function is evident in the elderly with more than two fall experiences. However, the groups showed no significant difference in maximum isometric knee extension, hip flexion, ankle extension, toe flexion strength, 10-meter maximum walking speed, functional reach, and one-legged standing duration with vision. Falling is considered to be a multiple risk factor syndrome related to many internal (i.e., disease and physical function) and external (i.e., bump and slippery floor) factors [23,24]. Although several fall risk factors have been outlined by the American Geriatric Society, falling rarely occurs because of a single factor. In most cases, multiple factors are involved simultaneously, although their relative levels differ. Therefore, the greater the number of related risk factors, the higher the fall incidence rate. Therefore, no significant difference was observed among the three groups with different fall experiences in the results of separate parameters.

A significant difference was observed among the three groups for ADL and fall risk scores. ADL scores for the group without fall experience were higher than those in the group with more than two fall experiences; the fall risk scores in the group with more than two fall experiences were higher than those in the other groups. ADL score measures the extent to which several daily living activities such as STS, gait, avoidance of obstacles and balance are performed. The fall risk score measures the increase in fall risk by disease, current medication dependency, a decrease in gait and balance abilities, muscle weakness, and external factors regarding the fall. In summary, both scores evaluate a plurality of factors simultaneously. Because many factors were evaluated by ADL and fall risk scores and were different from parameters such as 10-meter maximum walking speed, functional reach, and leg strength, they may have been able to discern the difference in fall experience more adequately. The first fall is often an accident. Moreover, elderly subjects with more than two fall experiences have markedly inferior physical functions than those without and with one fall experience $[25,26]$. Lord et al. [25] compared the physical functions between elderly subjects with and without fall experience and reported that visual function, sensory receptors, leg strength, reaction time, and balance functions were significantly inferior in elderly subjects with more than two fall experiences. Furthermore, Tucker et al. [26] and Lord et al. compared the Physiological Profile Assessment among three similar groups and reported that hand reaction time and sway on form were inferior in the group with more than two fall experiences. These results cumulatively suggest that a physical function test for fall prevention is necessary to evaluate the difference between elderly subjects with more than two fall experiences and those with one or no fall experience. However, even ADL and fall risk assessment scores, which measure multiple factors, were not able to completely establish this difference.

STS movement precedes ambulation and other basic ADL [14], and incorrectly performing this movement limits the independent life of the elderly [15]. We have indicated the importance of evaluating the ability to achieve STS movement and have thus developed an evaluation test $[18,27-30]$. STS movement is an upward transfer of the center of gravity movement $[16,17]$ and is divided into trunk flexion and trunk and knee extension phases. The former describes the phase between trunk flexion with forward (horizontal) transfer of the center of gravity from the initiation of movement until the hip lifts from the chair and the latter describes the phase between hip lift-off and a standing posture, with hips and both knees in extension. To achieve quick and smooth STS movement, the elderly require a coordinated ability to connect with the center of gravity transfer, which moves between the first and second phases, along with leg strength and balance ability beyond a certain level. In other words, multiple physical functions regarding the fall risk factors need to act in unison. Therefore, a complete evaluation of physical function with regard to fall occurrence is possible by measuring the ability to achieve STS movement. All three groups showed significant differences in maximum and mean center of gravity transfer velocity during STS movement. The elderly subjects without fall experience were the fastest and those with more than two fall experiences were the slowest. Therefore, the center of gravity transfer velocity during STS movement was considered useful for evaluating the total physical function with regard to falling.

\section{ACKNOWLEDGEMENTS}

The authors have no financial disclosures to make with regard to this paper. All authors contributed to all aspects of this paper, and no sponsor was involved in this study.

\section{REFERENCES}

[1] National Health and Medical Research Council (1994) Falls and the older person. Series on clinical management problems in the elderly. No. 6. Report of the health care committee expert panel for health care of the elderly. National Health and Medical Research Council, Australia.

[2] Lilley, J.M., Arie, T. and Chilvers, C.E. (1995) Accidents involving older people: A review of the literature. Age \& Ageing, 24, 346-365.

http://dx.doi.org/10.1093/ageing/24.4.346

[3] Demura, S., Sato, S., Yamaji, S., Kasuga, K. and Nagasawa, Y. (2011) Examination of validity of fall risk assessment items for screening high fall risk elderly among the healthy community-dwelling Japanese population. Ar- 
chives of Gerontology \& Geriatrics, 53, e41-e45. http://dx.doi.org/10.1016/j.archger.2010.10.010

[4] Demura, S., Sato, S., Shin, S. and Uchiyama, M. (2012) Setting the criterion for fall risk screening for healthy community-dwelling elderly. Archives of Gerontology \& Geriatrics, 54, 370-373.

http://dx.doi.org/10.1016/j.archger.2011.04.010

[5] Yasumura, S., Haga, H., Nagai, H., Shibata, H., Iwasaki, K., Ogawa, T., Ahiko, T. and Ihara, K. (1991) Incidence of and circumstances related to falls among the elderly in a Japanese community. Japanese Journal of Public Health, 38, 735-742.

[6] Niino, N. and Nakamura, K. (1996) Circumstances and factors related to falls in the institutionalized elderly. Japanese Journal of Geriatrics, 33, 12-16. http://dx.doi.org/10.3143/geriatrics.33.12

[7] Haga, H., Shibata, H., Shichita, K., Matsuzaki, T. and Hatano, S. (1986) Falls in the institutionalized elderly in Japan. Archives of Gerontology \& Geriatrics, 5, 1-9. http://dx.doi.org/10.1016/0167-4943(86)90002-6

[8] Kanis, J.A., Johnell, O. and De Laet, C. (2002) International variations in hip fracture probabilities: Implication for risk assessment. Journal of Bone Mineral Research, 17, 1237-1244.

http://dx.doi.org/10.1359/jbmr.2002.17.7.1237

[9] Kellogg International Work Group (1987) The prevention of falls in later life. A report of the Kellogg International Work Group on the prevention of falls by the elderly. Danish Medical Bulletin, 34, 1-24.

[10] Sattin, R.W., Lambert Huber, D.A., DeVito, C.A., Rodriguez, J.G., Ros, A., Bacchelli, S., Stevens, J.A. and Waxweiler, R.J. (1990) The incidence of fall injury events among the elderly in a defined population. American Journal of Epidemiology, 131, 1028-1037.

[11] Lilley, J.M., Arie, T. and Chilvers, C.E. (1995) Accidents involving older people: A review of the literature. Age \& Ageing, 24, 346-365.

http://dx.doi.org/10.1093/ageing/24.4.346

[12] Orimo, H. and Sakata, K. (2004) Results of fourth nationwide survey on femoral neck fracture. Japan Medical Journal, 4180, 25-30.

[13] American Geriatrics Society, British Geriatrics Society, American Academy of Orthopaedic Surgeons Panel on Falls Prevention (2001) Guideline for the prevention of falls in older persons. Journal of American Geriatric Society, 49, 664-672.

http://dx.doi.org/10.1046/j.1532-5415.2001.49115.x

[14] Riley, P.O., Schenkman, M.L., Mann, R.W. and Hodge, W.A. (1991) Mechanics of a constrained chair-rise. Journal of Biomechanics, 24, 77-85. http://dx.doi.org/10.1016/0021-9290(91)90328-K

[15] Alexander, N.B., Schultz, A.B. and Warwick, D.N. (1991) Rising from chair: Effect of age and functional ability on performance biomechanics. Journal of Gerontology, 46, M91-M98. http://dx.doi.org/10.1093/geronj/46.3.M91

[16] Vander Linden, D.W., Brunt, D. and McCulloch, M.U. (1994) Variant invariant characteristics of the sit-to-stand task in healthy elderly adults. Archives of Physical Medi- cine \& Rehabilitation, 75, 653-660. http://dx.doi.org/10.1016/0003-9993(94)90188-0

[17] Doorenbosch, C.A., Harlaar, J., Roebroeck, M.E. and Lankhorst, G.J. (1994) Two strategies of transferring from sit-to-stand; the activation of monoarticular and biarticular muscles. Journal of Biomechanics, 27, 1299-1307. http://dx.doi.org/10.1016/0021-9290(94)90039-6

[18] Yamada, T. and Demura, S. (2009) Reliability of center of gravity transfer velocity during the sit-to-stand movement and its relationship with leg muscle strength. Japan Journal of Test and Evaluation of Physical Education and Sports, 8, 33-38.

[19] Ebara, Y. and Yamamoto, S. (2001) Text of body dynamoics: Analysis of sit to stand movement. Ishiyaku Publishers, Japan, 1-4.

[20] Duncan, P.W., Weiner, D.K., Chandler, J. and Studenski, S. (1990) Functional reach: A new clinical measure of balance. Journal of Gerontology, 45, M192-M197. http://dx.doi.org/10.1093/geronj/45.6.M192

[21] Suzuki, T. (2000) Questionnaire for falls assessment of elderly people and its application. Health assessment manual. Kosei Kagaku Kenkyusho, Japan, 142-163.

[22] Schot, P.K., Knutzen, K.M., Poole, S.M. and Mrotek, L.A. (2003) Sit-to-stand performance of older adults following strength training. Research Quarterly for Exercise \& Sport, 74, 1-8.

http://dx.doi.org/10.1080/02701367.2003.10609058

[23] Cummings, S.R. (1996) Treatable and untreatable risk factors for hip fracture. Bone, 18, 165S-167S. http://dx.doi.org/10.1016/8756-3282(95)00505-6

[24] Gill, T.M. (1999) Preventing falls: To modify the environment or the individual? Journal of American Geriatric Society, 47, 1471-1472.

[25] Lord, S.R., Ward, J.A., Williams, P. and Anstey, K.J. (1994) Physiological factors associated with falls in older community-dwelling women. Journal of American Geriatric Society, 42, 1110-1117.

[26] Tucker, M.G., Kavanagh, J.J., Morrison, S. and Barrett, R.S. (2010) What are the relations between voluntary postural sway measures and falls-history status in community-dwelling older adults? Archives of Physical Medicine \& Rehabilitation, 91, 750-758. http://dx.doi.org/10.1016/j.apmr.2010.01.004

[27] Yamada, T. and Demura, S. (2005) Instruction in reliability and magnitude of evaluation parameters at each phase of a sit-to-stand movement. Perceptual \& Motor Skills, 101, 695-706.

[28] Demura, S. and Yamada, T. (2007) Height of chair seat and movement characteristics in sit-to-stand by young and elderly adults. Perceptual \& Motor Skills, 104, 21-31. http://dx.doi.org/10.2466/pms.104.1.21-31

[29] Yamada, T. and Demura, S. (2009) Relationships between ground reaction force parameters during a sit-to-stand movement and physical activity and falling risk of the elderly and a comparison of the movement characteristics between the young and the elderly. Archives of Gerontology \& Geriatrics, 48, 73-77.

http://dx.doi.org/10.1016/j.archger.2007.10.006 
[30] Yamada, T. and Demura, S. (2010) The relationship of force output characteristics during a sit-to-stand movement with lower limb muscle mass and knee joint exten- sion in the elderly. Archives of Gerontology \& Geriatrics, 50, e46-e50.

http://dx.doi.org/10.1016/j.archger.2009.05.009 